\title{
3,3-二甲基-1-(吡啶-3-基)-丁-2-酮肜酯的合成及抑菌活性
}

\author{
备宁宁 $a$ 张钰镭 $b$ 张 帅 $a$ 刘 思 $a$ 姜 林*, $a$ \\ ( ${ }^{a}$ 山东农业大学化学与材料科学学院 泰安 271018) \\ $\left({ }^{b}\right.$ 山东农业大学水利土木工程学院 泰安 271018)
}

\begin{abstract}
摘要 以 3-甲基吡啶、特戊酸乙酯、盐酸羟胺和取代苯甲酸等为原料, 经加成、肟化和酯化反应，合成了 12 种新型的 3,3-二甲基(吡啶-3-基)-丁-2-酮肟酯. 该方法反应条件比较温和、收率高. 化合物的结构通过 $\mathrm{IR} 、{ }^{1} \mathrm{H} \mathrm{NMR} 、{ }^{13} \mathrm{C} \mathrm{NMR}$ 和元素分析确证. 采用菌丝生长速率法测试了目标化合物对两种病原菌的离体抑制活性, $O$-(4-溴苯甲酰基)-3,3-二甲 基-1-(吡啶-3-基)-丁-2-酮肜(3h)和 $O$-(4-氯苯甲酰基)-3,3-二甲基-1-(吡啶-3-基)-丁-2-酮肜(3j)对菌核菌有较强的抑制活 性, $\mathrm{EC}_{50}$ 值分别为 5.07 和 $4.81 \mu \mathrm{g} / \mathrm{mL} ; \mathbf{3 h} 、 3 \mathbf{j}$ 和 $O$-(3,4-二氯苯甲酰基)-3,3-二甲基-1-(吡啶-3-基)-丁-2- 酮肜(3I)对灰霉菌 显示出较高的抑制活性, $\mathrm{EC}_{50}$ 值分别为 $4.98 、 5.44$ 和 $6.34 \mu \mathrm{g} / \mathrm{mL}$.
\end{abstract}

关键词 吡啶; 酮; 肜; 羧酸; 合成设计; 抑菌活性

\section{Synthesis of Novel 3,3-Dimethyl-1-(pyridin-3-yl)butan-2-one Oxime Esters and Evaluation of Their Antifungal Activity}

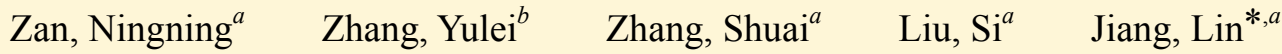 \\ ( ${ }^{a}$ College of Chemistry and Material Science, Shandong Agricultural University, Taian 271018) \\ ( ${ }^{b}$ College of Water Conservancy and Civil Engineering, Shandong Agricultural University, Taian 271018)
}

\begin{abstract}
A series of novel pyridine derivatives containing oxime esters have been synthesized from 3-methylpyridine, ethy pivalate, hydroxylamine hydrochloride and substituted benzoic acid by addition, oximization and esterification reactions, which has the advantages of moderate reaction condition and high yield. Their structures were elucidated by IR, ${ }^{1} \mathrm{H} N \mathrm{NR},{ }^{13} \mathrm{C}$ NMR and elemental analysis. Moreover, the target compounds were evaluated for their antifungal activity against two plant pathogens by the mycelium growth rate method. The results indicated that $O$-(4-bromobenzoyl)-3,3-dimethyl-1-(pyridin-3yl)butan-2-one oxime (3h) and $O$-(4-chlorobenzoyl)-3,3-dimethyl-1-(pyridin-3-yl)butan-2-one oxime (3j) exhibited strong antifungal activity against $S$. sclerotiorum with $\mathrm{EC}_{50}$ values of 5.07 and $4.81 \mu \mathrm{g} / \mathrm{mL}$, and $\mathbf{3 h}, \mathbf{3} \mathbf{j}$ and $O-(3,4$-dichlorobenzoyl)3,3-dimethyl-1-(pyridin-3-yl)butan-2-one oxime (3I) displayed high antifungal activity against $B$. cinerea with $\mathrm{EC}_{50}$ values of $4.98,5.44$ and $6.34 \mu \mathrm{g} / \mathrm{mL}$, respectively.

Keywords pyridine; ketones; oxime; carboxylic acids; synthesis design; antifungal activity
\end{abstract}

吡啶衍生物具有优良和广谱的生物活性，如抑

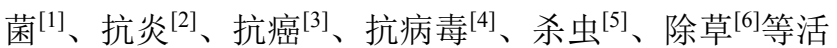
性，广泛应用于农药和医药领域. 另一方面，吡啶是苯 的电子等排体, 但其疏水性常数比苯低得多, 因此吡啶 衍生物往往具有活性更高、毒性低、内吸性强、选择性 好等优点 ${ }^{[7]}$. 最近十几年来已开发出 18 种含吡啶结构的 农药品种 ${ }^{[7]}$, 其中包括高效杀菌剂啶斑肟、啶氧菌酯、 啶酰菌胺等.

肜类化合物(肜醚和肜酯)具有杀菌 ${ }^{[8]}$ 、抗炎 ${ }^{[9]}$ 、抗病

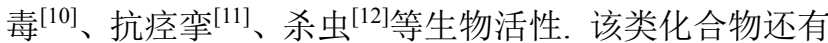
高效、低毒、低残留等特点，作为一类农药和医药活性 物质得到较广泛的应用. 目前农药市场中, 肜类农药主 要是含肜醚结构的化合物, 如杀菌剂啶斑肜、肜菌酯、 肜醚菌胺，除草剂肜草酮，杀虫剂肜醚菊酯等，但鲜有 肟酯类农药品种. 为发现高活性的肟酯类杀菌剂, 许多 化学工作者开展了很有价值的研究. 例如, 2012 年, Naik 等 ${ }^{[13]}$ 设计合成了一系列含香兰素结构的吡啶酮 肜酯，其中 2 个化合物对黄曲霉菌、金孢子菌、白色念

\footnotetext{
* Corresponding author(s). E-mail: jiangl@sdau.edu.cn

Received May 5, 2017; revised June 1, 2017; published online June 16, 2017.

Project supported by the Natural Science Foundation of Shandong Province (No. ZR2014BM030).

山东省自然科学基金(No. ZR2014BM030)资助项目.
} 
珠菌有很好的抑制作用, 与对照药剂氟康唑活性相当.

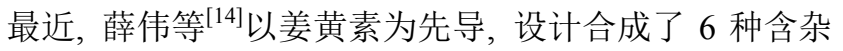
环的戊二烯酮肜酯, 其中 4 种化合物对小麦赤霉病菌、 苹果腐烂病菌有中等抑制活性. 为寻找具有优良杀菌活 性的含吡啶结构的肟酯类化合物, 本文以啶斑肜为先导 化合物, 再结合三唑类杀菌剂三唑酮的结构, 设计合成 了 12 种结构新颖的 3,3-二甲基-1-(吡啶-3-基)-丁-2-酮肜 酯 $(3 \mathbf{3} \sim 31)$, 并初步测试了目标化合物的对灰霉菌、菌核 菌的抑制活性. 设计思路见 Scheme 1.

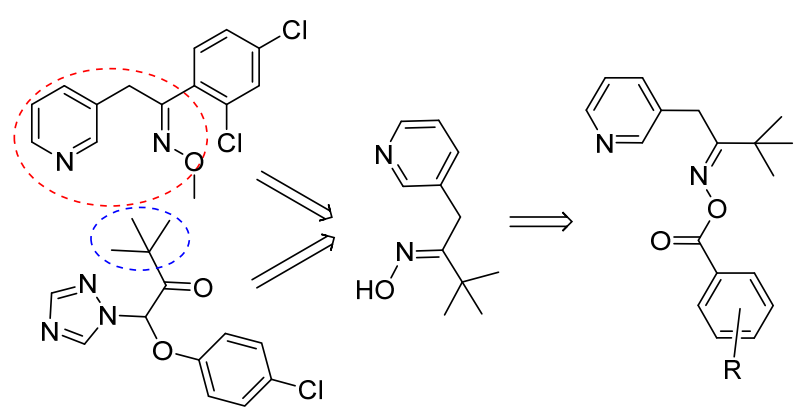

图式 1 目标化合物的设计思路

Scheme 1 Design strategy of the target compounds

\section{1 结果与讨论}

\section{1 合成与波谱}

3-甲基吡啶在强碱二异丙基氨基锂(LDA)作用下转 化为 3-吡啶甲基锂, 后者与特戊酸乙酯加成生成 3,3-二 甲基-1-(吡啶-3-基)-丁-2-酮(1), 1 在碱性介质中与盐酸 羟胺反应得到中间体酮肪 (2). 最后, 该酮肜与取代苯甲 酸在 DCC/HOBt 作用下直接缩合生成目标化合物 3(合 成路线见 Scheme 2). 在吡啶甲基锂的制备中, 文献报 道反应温度一般在 $-30 \sim-78{ }^{\circ} \mathrm{C}^{[15]}$, 但是在本实验中, 我们发现温度可以上升至 $-10 \sim-15^{\circ} \mathrm{C}$, 使操作更加方 便. 此外, 我们采用肜与取代苯甲酸在 $\mathrm{DCC} / \mathrm{HOBt}$ 催化 下直接合成目标化合物，收率在 $90 \% \sim 95 \%$ 之间.

在 ${ }^{1} \mathrm{H}$ NMR 谱中, 化学位移 $\delta$ 在 $1.25 \sim 1.31$ 之间的 单峰为叔丁基上甲基质子的共振吸收, $\delta$ 在 3.89 3.93 的单峰是与吡啶环相连的亚甲基质子信号, 而苯环和吡 啶环上质子的共振吸收出现在 $\delta 6.87 \sim 8.54$ 处. ${ }^{13} \mathrm{C}$ NMR 中, 羰基的共振吸收出现在 $\delta 171.8 \sim 172.9$ 处, 叔 丁基季碳的信号出现在 $\delta 38$ 附近. IR 谱中, 在 1746 $1758 \mathrm{~cm}^{-1}$ 之间的强峰为酯羰基的伸缩振动, 而 1605 $1622 \mathrm{~cm}^{-1}$ 处的中等强度的峰属于 $\mathrm{C}=\mathrm{N}$ 和 $\mathrm{C}=\mathrm{C}$ 伸缩振 动, 肜酯基 $\mathrm{N}-\mathrm{O}-\mathrm{C}$ 的伸缩振动吸收位于 1241 1286 $\mathrm{cm}^{-1}$ 之间.

\section{2 抑菌活性}

由表 1 可见, 目标化合物 $\mathbf{3 h}$ 和 $\mathbf{3 j}$ 对菌核菌有较强
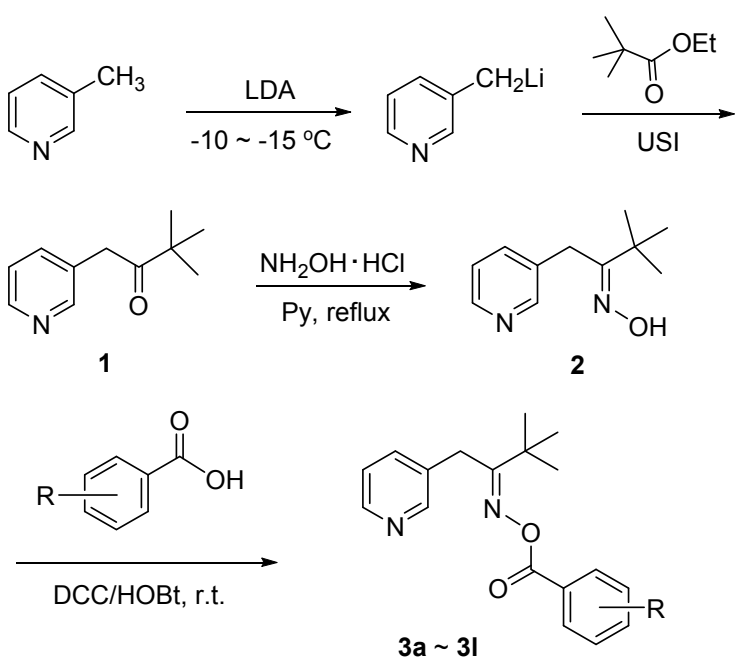

图式 2 目标化合物 $3 a \sim 31$ 的合成路线

Scheme 2 Synthetic route for the target compounds $\mathbf{3 a} \sim 3 \mathbf{3}$

的抑制活性, $\mathrm{EC}_{50}$ 值分别为 5.07 和 $4.81 \mu \mathrm{g} / \mathrm{mL}$, 其活性 明显高于商品化杀菌剂三唑酮 $\left(\mathrm{EC}_{50}=11.69 \mu \mathrm{g} / \mathrm{mL}\right)$, 但 低于百菌清 $\left(E_{50}=3.52 \mu \mathrm{g} / \mathrm{mL}\right)$. 另一方面, $3 \mathbf{h}$ 和 $3 \mathbf{j}$ 对 灰霉菌显示出良好的抑制活性, $\mathrm{EC}_{50}$ 值分别为 4.98 和 $5.44 \mu \mathrm{g} / \mathrm{mL}$, 其活性高于百菌清 $\left(\mathrm{EC}_{50}=6.28 \mu \mathrm{g} / \mathrm{mL}\right) ; 31$ 对灰霉菌也有较高的抑制活性, $\mathrm{EC}_{50}$ 值为 $6.34 \mu \mathrm{g} / \mathrm{mL}$, 表明活性与百菌清相近; 这三种化合物的活性均高于三 唑酮 $\left(\mathrm{EC}_{50}=10.41 \mu \mathrm{g} / \mathrm{mL}\right)$.

通过分析化合物的抑菌活性，发现苯环中取代基的 类型和位置对活性有显著影响. 取代基处于苯环对位 时，化合物的抑菌活性高于在邻位或间位的类似物(例 如 $3 b \sim 3 d, 3 e \sim 3 g$ 和 $3 i \sim 3 j$ ). 总体来看, 苯环对位含有 $\mathrm{Cl}$ 或 $\mathrm{Br}$ 原子的化合物具有较好的抑菌活性.

\section{2 结论}

以 3-甲基吡啶、特戊酸乙酯、盐酸差胺和取代苯甲 酸等为原料, 经加成、肜化和酯化等三步反应，合成了 12 种新型的 3,3-二甲基-1-(吡啶-3-基)-丁-2-酮肜酯. 该 方法反应条件比较温和、收率高. 测试了目标化合物对 菌核菌、灰霉菌的离体抑制活性, 获得几种对菌核菌和 灰霉菌有较高抑制的化合物, 为开展具有杀菌活性的吡 啶类化合物的设计、合成提供了有益参考.

\section{3 实验部分}

\section{1 仪器与试剂}

北京泰克仪器有限公司 X-5 精密显微熔点测定仪 (温度计未校正); 美国 Nicolet 380 型傅里叶变换红外光 谱仪 ( $\mathrm{KBr}$ 压片法); 美国 Mercury Plus-400型核磁共振仪 $\left(\mathrm{CDCl}_{3}\right.$ 为溶剂, $\mathrm{TMS}$ 为内标); 德国 Elementar 公司 Vario 
表 1 化合物 3a $~ 31$ 的抑菌活性

Table 1 Antifungal activity for the target compounds $\mathbf{3 a} \sim 3 \mathbf{I}$

\begin{tabular}{clll}
\hline \multirow{2}{*}{ Compd. } & $\mathrm{R}$ & \multicolumn{1}{c}{$\mathrm{EC}_{50}{ }^{a} /\left(\mu \mathrm{g} \cdot \mathrm{mL}^{-1}\right)$} \\
\cline { 3 - 4 } & & \multicolumn{1}{c}{ S. sclerotiorum } & B. cinerea \\
\hline $\mathbf{3 a}$ & $\mathrm{H}$ & $24.62(18.82 \sim 32.20)$ & $64.59(51.91 \sim 80.35)$ \\
$\mathbf{3 b}$ & $2-\mathrm{CH}_{3}$ & $42.09(34.88 \sim 50.80)$ & $46.44(43.61 \sim 49.43)$ \\
$\mathbf{3 c}$ & $3-\mathrm{CH}_{3}$ & $18.83(16.91 \sim 20.95)$ & $37.74(31.08 \sim 45.83)$ \\
$\mathbf{3 d}$ & $4-\mathrm{CH}_{3}$ & $15.77(15.18 \sim 16.39)$ & $8.00(6.99 \sim 9.17)$ \\
$\mathbf{3 e}$ & $2-\mathrm{CH}_{3} \mathrm{O}$ & $62.02(59.00 \sim 65.19)$ & $71.78(64.60 \sim 79.76)$ \\
$\mathbf{3 f}$ & $3-\mathrm{CH}_{3} \mathrm{O}$ & $25.13(20.85 \sim 30.29)$ & $67.13(62.18 \sim 72.46)$ \\
$\mathbf{3 g}$ & $4-\mathrm{CH}_{3} \mathrm{O}$ & $11.89(11.15 \sim 12.69)$ & $35.32(34.09 \sim 36.59)$ \\
$\mathbf{3 h}$ & $4-\mathrm{Br}$ & $5.07(4.31 \sim 5.98)$ & $4.98(4.55 \sim 5.44)$ \\
$\mathbf{3 i}$ & $2-\mathrm{Cl}$ & $43.80(32.69 \sim 58.68)$ & $52.51(41.48 \sim 66.46)$ \\
$\mathbf{3 j}$ & $4-\mathrm{Cl}$ & $4.81(3.87 \sim 5.97)$ & $5.44(5.08 \sim 5.83)$ \\
$\mathbf{3 k}$ & $2,4-\mathrm{Cl}$ & $25.80(21.91 \sim 30.37)$ & $46.92(38.82 \sim 56.69)$ \\
$\mathbf{3 l}$ & $3,4-\mathrm{Cl}$ & $9.51(7.19 \sim 12.60)$ & $6.34(5.64 \sim 7.13)$ \\
Triadimefon & & $11.69(10.85 \sim 12.61)$ & $10.41(8.22 \sim 13.19)$ \\
Chlorothalonil & & $3.52(2.63 \sim 4.90)$ & $6.28(4.32 \sim 8.05)$ \\
\hline
\end{tabular}

${ }^{a}$ 95\% CL: 95\% confidence interval.

EL 型元素分析仪; GXZ 型智能光照培养箱. 试剂为市售 分析纯或化学纯.

\section{2 实验方法}

\subsubsection{3,3-二甲基-1-(吡啶-3-基)-丁-2-酮(1)的合成}

在装有 $\mathrm{N}_{2}$ 保护装置、冰盐浴冷却的 $50 \mathrm{~mL}$ 二颈烧 瓶中, 加入二异丙基氨基锂 $\left(10 \mathrm{~mL}, 2 \mathrm{~mol} \cdot \mathrm{L}^{-1}\right.$ 庚烷/四氢 呋喃/乙苯溶液), 待温度降至 $-10 \sim-15^{\circ} \mathrm{C}$, 电磁搅拌 下向瓶中缓慢滴加 3-甲基吡啶(1.88 g, $20 \mathrm{mmol}$ ), 继续 搅拌 $1 \mathrm{~h}$. 在此温度下缓缓滴加特戊酸乙酯 $(2.60 \mathrm{~g}, 20$ $\mathrm{mmol})$ 后, 升至室温, 并将反应混合物置于超声波反应 器(300 W)反应 $30 \mathrm{~min}$, 加水 $(10 \mathrm{~mL})$ 淬灭反应, 用乙酸 乙酯提取 $(20 \mathrm{~mL} \times 2)$. 有机相用无水 $\mathrm{Na}_{2} \mathrm{SO}_{4}$ 干燥, 减压 脱溶剂得粗产物. 以石油醚/乙酸乙酯 $(V: V=3: 1)$ 为 洗脱剂, 硅胶柱层析进行纯化得到化合物 1 , 浅黄色油 状物, 产率 $85 \%$. ${ }^{1} \mathrm{H} \mathrm{NMR}\left(\mathrm{CDCl}_{3}, 400 \mathrm{MHz}\right) \delta: 1.30[\mathrm{~s}$, $\left.9 \mathrm{H}, \mathrm{C}\left(\mathrm{CH}_{3}\right)_{3}\right], 3.81\left(\mathrm{~s}, 2 \mathrm{H}, \mathrm{CH}_{2}\right), 7.24 \sim 7.27(\mathrm{~m}, 1 \mathrm{H}$, Py-H), 7.54 (d, J=4.0 Hz, 1H, Py-H), 8.41 (s, 1H, Py-H), 8.55 (d, $J=4.0 \mathrm{~Hz}, 1 \mathrm{H}, \mathrm{Py}-\mathrm{H})$; IR (KBr) v: 2954, 1689 $\mathrm{cm}^{-1}$. Anal. calcd for $\mathrm{C}_{11} \mathrm{H}_{15} \mathrm{NO}$ : C 74.54, H 8.53, N 7.90; found C 74.46, H 8.34, N 7.87.

3.2.2 3,3-二甲基-1-(吡啶-3-基)-丁-2-酮肜(2)的合成

$25 \mathrm{~mL}$ 烧瓶中加入化合物 1 (1.77 g, $10 \mathrm{mmol})$ 、盐酸 羟胺 $(0.70 \mathrm{~g}, 10 \mathrm{mmol})$ 和 $3 \mathrm{~mL}$ 吡啶. 将反应混合物置于 $80^{\circ} \mathrm{C}$ 的水浴中加热搅拌 $3 \mathrm{~h}$, 反应完毕, 减压蒸除吡啶, 残余物用乙酸乙酯提取 $(20 \mathrm{~mL})$, 无水 $\mathrm{Na}_{2} \mathrm{SO}_{4}$ 干燥, 旋 蒸除去溶剂. 粗产物经柱层析提纯 [石油醚/乙酸乙酯 $(V: V=5: 1)]$, 得酮肜 2. 白色固体, 产率 $89 \%$. m.p. $121.2 \sim 122.3{ }^{\circ} \mathrm{C} ;{ }^{1} \mathrm{H}$ NMR $\left(\mathrm{CDCl}_{3}, 400 \mathrm{MHz}\right) \delta: 1.13[\mathrm{~s}$, $\left.9 \mathrm{H}, \mathrm{C}\left(\mathrm{CH}_{3}\right)_{3}\right], 3.79\left(\mathrm{~s}, 2 \mathrm{H}, \mathrm{CH}_{2}\right), 7.20 \sim 8.53(\mathrm{~m}, 4 \mathrm{H}$,
Py-H), 9.15 (s, 1H, OH); IR (KBr) v: 3170, $1636 \mathrm{~cm}^{-1}$. Anal. calcd for $\mathrm{C}_{19} \mathrm{H}_{22} \mathrm{~N}_{2} \mathrm{O}_{2}: \mathrm{C}$ 68.72, $\mathrm{H}$ 8.39, N 14.57; found C 68.46, H 8.24, N 14.87.

3.2.3 目标化合物 3,3-二甲基-1-(吡啶-3-基)-丁-2-酮 肜酯(3) 的合成

$25 \mathrm{~mL}$ 烧瓶中加入酮肟 $2(0.59 \mathrm{~g}, 3 \mathrm{mmol})$ 、取代苯 甲酸 $(3 \mathrm{mmol})$, 用 $10 \mathrm{~mL}$ 干燥的二氯甲烷溶解, 然后加 入二环已基碳二亚胺( DCC, $0.62 \mathrm{~g}, 3 \mathrm{mmol}$ )和 1-差基苯 并三唑(HOBt, $0.41 \mathrm{~g}, 3 \mathrm{mmol})$. 反应混合液室温下摚拌 $2 \mathrm{~h}$. 反应完毕, 过滤除去固体, 滤液脱溶剂得粗产物. 以石油醚/乙酸乙酯 $(V: V=3: 1)$ 为洗脱剂, 硅胶柱层 析进行纯化得目标化合物 $\mathbf{3 a} \sim \mathbf{3 1}$.

$O$-苯甲酰基-3,3-二甲基-1-(吡啶-3-基)-丁-2-酮肜 (3a): 白色固体，产率 91\%. m.p. 79.8 80.5 ${ }^{\circ} \mathrm{C} ;{ }^{1} \mathrm{H}$ NMR $\left(400 \mathrm{MHz}, \mathrm{CDCl}_{3}\right) \delta: 1.29\left[\mathrm{~s}, 9 \mathrm{H}, \mathrm{C}\left(\mathrm{CH}_{3}\right)_{3}\right], 3.94(\mathrm{~s}, 2 \mathrm{H}$, $\left.\mathrm{CH}_{2}\right), 7.21 \sim 7.75$ (m, 7H, Py-H+Ph-H), 8.47 (d, $J=3.6$ $\mathrm{Hz}, 1 \mathrm{H}, \mathrm{Py}-\mathrm{H}), 8.55$ (s, 1H, Py-H); ${ }^{13} \mathrm{C}$ NMR (100 MHz, $\left.\mathrm{CDCl}_{3}\right) \delta: 172.3,163.6,149.6,147.9,135.1,133.2,131.7$, 129.3, 128.8, 128.5, 123.6, 38.9, 30.5, 27.8; IR (KBr) v: 1739, 1615, $1248 \mathrm{~cm}^{-1}$. Anal. calcd for $\mathrm{C}_{18} \mathrm{H}_{20} \mathrm{~N}_{2} \mathrm{O}_{2}$ : C 72.95, H 6.80, N 9.45; found C 72.85, H 6.85, N 9.75.

$O$-(2-甲基苯甲酰基)-3,3-二甲基-1-(吡啶-3-基)-丁2-酮肪(3b): 浅黄色油状物, 产率 $94 \%$. [n $]_{\mathrm{D}}^{20} 1.5728 .{ }^{1} \mathrm{H}$ NMR (400 MHz, $\left.\mathrm{CDCl}_{3}\right) \delta: 1.29\left[\mathrm{~s}, 9 \mathrm{H}, \mathrm{C}\left(\mathrm{CH}_{3}\right)_{3}\right], 2.54(\mathrm{~s}$, $\left.3 \mathrm{H}, \mathrm{CH}_{3}\right), 3.90\left(\mathrm{~s}, 2 \mathrm{H}, \mathrm{CH}_{2}\right), 7.07 \sim 7.49(\mathrm{~m}, 6 \mathrm{H}, \mathrm{Py}-\mathrm{H}+$ $\mathrm{Ph}-\mathrm{H}), 8.47$ (d, J=3.2 Hz, 1H, Py-H), 8.51 (s, 1H, Py-H); ${ }^{13} \mathrm{C}$ NMR $\left(100 \mathrm{MHz}, \mathrm{CDCl}_{3}\right) \delta: 171.9,164.4,149.5,147.7$, $140.7,135.3,132.2,131.8,131.7,129.8,128.0,125.7$, 123.6, 38.8, 30.6, 27.9, 21.5; IR (KBr) v: 1741, 1615, 1245 
$\mathrm{cm}^{-1}$. Anal. calcd for $\mathrm{C}_{19} \mathrm{H}_{22} \mathrm{~N}_{2} \mathrm{O}_{2}:$ C 73.52, H 7.14, N 9.03; found C 73.36, H 7.24, N 8.82.

$O$-(3-甲基苯甲酰基)-3,3-二甲基-1-(吡啶-3-基)-丁2-酮肜(3c): 白色固体, 产率 90\%. m.p. $62.2 \sim 62.8{ }^{\circ} \mathrm{C}$; ${ }^{1} \mathrm{H}$ NMR (400 MHz, $\left.\mathrm{CDCl}_{3}\right) \delta: 1.30\left[\mathrm{~s}, 9 \mathrm{H}, \mathrm{C}\left(\mathrm{CH}_{3}\right)_{3}\right], 2.30$ (s, $\left.3 \mathrm{H}, \mathrm{CH}_{3}\right), 3.93\left(\mathrm{~s}, 2 \mathrm{H}, \mathrm{CH}_{2}\right), 7.20 \sim 7.52(\mathrm{~m}, 6 \mathrm{H}, \mathrm{Py}-$ $\mathrm{H}+\mathrm{Ph}-\mathrm{H}), 8.48(\mathrm{~d}, J=3.2 \mathrm{~Hz}, 1 \mathrm{H}, \mathrm{Py}-\mathrm{H}), 8.55$ (s, $1 \mathrm{H}$, Py-H); ${ }^{13} \mathrm{C}$ NMR $\left(100 \mathrm{MHz}, \mathrm{CDCl}_{3}\right) \delta: 172.1,163.8$, $149.5,147.8,138.3,135.1,134.0,131.8,129.9,128.7$, 128.4, 126.5, 123.6, 38.9, 30.6, 27.8, 21.2; IR (KBr) v: $1741,1615,1265 \mathrm{~cm}^{-1}$. Anal. calcd for $\mathrm{C}_{19} \mathrm{H}_{22} \mathrm{~N}_{2} \mathrm{O}_{2}$ : C 73.52, H 7.14, N 9.03; found C 73.61, H 7.25, N 9.15.

$O$-(4-甲基苯甲酰基)-3,3-二甲基-1-(吡啶-3-基)-丁2-酮肪(3d): 白色固体, 产率 93\%. m.p. 99.7 100.5 ${ }^{\circ} \mathrm{C}$; ${ }^{1} \mathrm{H} \mathrm{NMR}\left(400 \mathrm{MHz}, \mathrm{CDCl}_{3}\right) \delta: 1.29\left[\mathrm{~s}, 9 \mathrm{H}, \mathrm{C}\left(\mathrm{CH}_{3}\right)_{3}\right], 2.36$ (s, $\left.3 \mathrm{H}, \mathrm{CH}_{3}\right), 3.92\left(\mathrm{~s}, 2 \mathrm{H}, \mathrm{CH}_{2}\right), 7.13 \sim 7.63(\mathrm{~m}, 6 \mathrm{H}, \mathrm{Py}-\mathrm{H}$ $+\mathrm{Ph}-\mathrm{H}), 8.47$ (d, $J=3.2 \mathrm{~Hz}, 1 \mathrm{H}, \mathrm{Py}-\mathrm{H}), 8.55(\mathrm{~s}, 1 \mathrm{H}$, Py-H); ${ }^{13} \mathrm{C}$ NMR $\left(100 \mathrm{MHz}, \mathrm{CDCl}_{3}\right) \delta: 172.0,163.7$, $149.5,147.8,144.0,135.1,131.8,129.4,129.2,125.9$, 123.6, 38.8, 30.5, 27.8, 21.7; IR (KBr) v: 1731, 1610, 1261 $\mathrm{cm}^{-1}$. Anal. calcd for $\mathrm{C}_{19} \mathrm{H}_{22} \mathrm{~N}_{2} \mathrm{O}_{2}:$ C 73.52, H 7.14, N 9.03; found C 73.46, H 7.34, N 8.87.

$O$-(2-甲氧基苯甲酰基)-3,3-二甲基-1-(吡啶-3-基)丁-2-酮肜(3e): 白色固体, 产率 92\%. m.p. 56.2 $56.8{ }^{\circ} \mathrm{C} ;{ }^{1} \mathrm{H}$ NMR $\left(400 \mathrm{MHz}, \mathrm{CDCl}_{3}\right) \delta: 1.25[\mathrm{~s}, 9 \mathrm{H}$, $\left.\mathrm{C}\left(\mathrm{CH}_{3}\right)_{3}\right], 3.62\left(\mathrm{~s}, 3 \mathrm{H}, \mathrm{OCH}_{3}\right), 3.92\left(\mathrm{~s}, 2 \mathrm{H}, \mathrm{CH}_{2}\right), 6.87 \sim$ 7.61 (m, 6H, Py-H+Ph-H), 8.45 (d, $J=4.4 \mathrm{~Hz}, 1 \mathrm{H}, \mathrm{Py}-\mathrm{H})$, 8.52 (s, $1 \mathrm{H}, \mathrm{Py}-\mathrm{H}) ;{ }^{13} \mathrm{C}$ NMR $\left(100 \mathrm{MHz}, \mathrm{CDCl}_{3}\right) \delta: 172.1$, $164.2,158.8,149.8,147.7,135.5,133.8,131.9,123.4$, $120.2,118.8,111.7,110.0,55.5,38.8,30.6,28.0$; IR (KBr) v: $1738,1615,1286 \mathrm{~cm}^{-1}$. Anal. calcd for $\mathrm{C}_{19} \mathrm{H}_{22} \mathrm{~N}_{2} \mathrm{O}_{3}: \mathrm{C}$ 69.92, H 6.79, N 8.58; found C 69.75, H 6.62, N 8.64.

$O$-(3-甲氧基苯甲酰基)-3,3-二甲基-1-(吡啶-3-基)丁-2-酮肜(3f): 白色固体, 产率 92\%. m.p. 49.7 $50.8{ }^{\circ} \mathrm{C} ;{ }^{1} \mathrm{H}$ NMR $\left(400 \mathrm{MHz}, \mathrm{CDCl}_{3}\right) \delta: 1.29[\mathrm{~s}, 9 \mathrm{H}$, $\left.\mathrm{C}\left(\mathrm{CH}_{3}\right)_{3}\right], 3.71\left(\mathrm{~s}, 3 \mathrm{H}, \mathrm{OCH}_{3}\right), 3.93\left(\mathrm{~s}, 2 \mathrm{H}, \mathrm{CH}_{2}\right), 7.03 \sim$ 7.48 (m, 6H, Py-H+Ph-H), 8.46 (d, $J=4.8$ Hz, 1H, Py-H), 8.54 (s, 1H, Py-H); ${ }^{13} \mathrm{C}$ NMR (100 MHz, $\left.\mathrm{CDCl}_{3}\right) \delta: 172.2$, $163.5,159.5,149.5,147.9,135.0,131.6,130.1,129.5$, 123.6, 121.7, 119.8, 113.7, 55.3, 38.9, 30.5, 27.8; IR (KBr) v: $1743,1615,1272 \mathrm{~cm}^{-1}$. Anal. calcd for $\mathrm{C}_{19} \mathrm{H}_{22} \mathrm{~N}_{2} \mathrm{O}_{3}: \mathrm{C}$ 69.92, H 6.79, N 8.58; found C 69.74, H 6. 70, N 8.44\%.

$O$-(4-甲氧基苯甲酰基)-3,3-二甲基-1-(吡啶-3-基)丁-2-酮肜 (3g): 白色固体, 产率 92\%. m.p. 58.5 $60.1{ }^{\circ} \mathrm{C} ;{ }^{1} \mathrm{H}$ NMR $\left(400 \mathrm{MHz}, \mathrm{CDCl}_{3}\right) \delta: 1.28[\mathrm{~s}, 9 \mathrm{H}$,
$\left.\mathrm{C}\left(\mathrm{CH}_{3}\right)_{3}\right], 3.82\left(\mathrm{~s}, 3 \mathrm{H}, \mathrm{CH}_{3}\right), 3.92\left(\mathrm{~s}, 2 \mathrm{H}, \mathrm{CH}_{2}\right), 6.81 \sim 7.70$ (m, 6H, Py- $\mathrm{H}+\mathrm{Ph}-\mathrm{H}), 8.47$ (d, $J=3.6 \mathrm{~Hz}, 1 \mathrm{H}, \mathrm{Py}-\mathrm{H})$, 8.55 (s, $1 \mathrm{H}, \mathrm{Py}-\mathrm{H}) ;{ }^{13} \mathrm{C}$ NMR $\left(100 \mathrm{MHz}, \mathrm{CDCl}_{3}\right) \delta: 171.8$, $163.5,163.4,149.6,147.8,135.1,131.9,131.4,123.6$, 121.0, 113.7, 55.4, 38.8, 30.5, 27.8; IR (KBr) $v: 1739$, $1605,1251 \mathrm{~cm}^{-1}$. Anal. calcd for $\mathrm{C}_{19} \mathrm{H}_{22} \mathrm{~N}_{2} \mathrm{O}_{3}: \mathrm{C} 69.92, \mathrm{H}$ 6.79, N 8.58; found C 69.66, H 6.53, N 8.64.

$O$-(4-溴苯甲酰基)-3,3-二甲基-1-(吡啶-3-基)-丁-2酮肜(3h): 浅黄色固体, 产率 $91 \%$. m.p. $88.2 \sim 89.6{ }^{\circ} \mathrm{C}$;

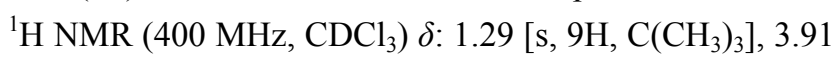
(s, $\left.2 \mathrm{H}, \mathrm{CH}_{2}\right), 7.21 \sim 7.56(\mathrm{~m}, 6 \mathrm{H}, \mathrm{Py}-\mathrm{H}+\mathrm{Ph}-\mathrm{H}), 8.48(\mathrm{~d}$, $J=3.2 \mathrm{~Hz}, 1 \mathrm{H}, \mathrm{Py}-\mathrm{H}), 8.53$ (s, 1H, Py-H); ${ }^{13} \mathrm{C}$ NMR $(100$ $\left.\mathrm{MHz}, \mathrm{CDCl}_{3}\right) \delta: 172.5,163.0,149.5,148.0,135.0,131.9$, 131.6, 130.8, 128.4, 127.7, 123.6, 38.9, 30.6, 27.8; IR (KBr): 1746, 1621, $1248 \mathrm{~cm}^{-1}$. Anal. calcd for $\mathrm{C}_{18} \mathrm{H}_{19} \mathrm{Br}-$ $\mathrm{N}_{2} \mathrm{O}_{2}$ : C 57.61, H 5.10, N 7.47; found C 57.43, H 5.12, N $7.57 \%$.

$O$-(2-氯苯甲酰基)-3,3-二甲基-1-(吡啶-3-基)-丁-2酮肜(3i): 白色固体, 产率 91\%. m.p. 94.6 95.2 ${ }^{\circ} \mathrm{C} ;{ }^{1} \mathrm{H}$ NMR (400 MHz, $\left.\mathrm{CDCl}_{3}\right) \delta: 1.27\left[\mathrm{~s}, 9 \mathrm{H}, \mathrm{C}\left(\mathrm{CH}_{3}\right)_{3}\right], 3.90$ (s, $\left.2 \mathrm{H}, \mathrm{CH}_{2}\right), 7.18 \sim 7.47$ (m, 6H, Py-H+Ph-H), 8.45 (d, $J=$ $4.8 \mathrm{~Hz}, 1 \mathrm{H}, \mathrm{Py}-\mathrm{H}), 8.47$ (s, 1H, Py-H); ${ }^{13} \mathrm{C}$ NMR (100 $\left.\mathrm{MHz}, \mathrm{CDCl}_{3}\right) \delta: 172.7,163.2,149.6,147.8,135.2,133.2$, 132.7, 131.6, 131.0, 130.9, 129.2, 126.6, 123.5, 38.9, 30.7, 27.9; IR (KBr) v: 1750, 1610, $1241 \mathrm{~cm}^{-1}$. Anal. calcd for $\mathrm{C}_{18} \mathrm{H}_{19} \mathrm{ClN}_{2} \mathrm{O}_{2}$ : C 65.35, H 5.79, N 8.47; found C 65.43, H $5.89, \mathrm{~N} 8.57$.

$O$-(4-氯苯甲酰基)-3,3-二甲基-1-(吡啶-3-基)-丁-2酮肜(3j): 白色固体, 产率 95\%. m.p. 97.4 98.0 ${ }^{\circ} \mathrm{C} ;{ }^{1} \mathrm{H}$ NMR (400 MHz, $\left.\mathrm{CDCl}_{3}\right) \delta: 1.30\left[\mathrm{~s}, 9 \mathrm{H}, \mathrm{C}\left(\mathrm{CH}_{3}\right)_{3}\right], 3.93$ (s, $2 \mathrm{H}, \mathrm{CH}_{2}$ ), $7.21 \sim 7.64$ (m, 6H, Py-H+Ph-H), 8.48 (d, $J=$ $4.8 \mathrm{~Hz}, 1 \mathrm{H}, \mathrm{Py}-\mathrm{H}), 8.54$ (s, 1H, Py-H); ${ }^{13} \mathrm{C}$ NMR $(100$ $\left.\mathrm{MHz}, \mathrm{CDCl}_{3}\right) \delta: 172.5,162.8,149.5,147.9,139.7,135.0$, $131.6,130.7,128.9,127.2,123.6,38.9,30.6,27.8$; IR (KBr) $v$ : 1746, 1622, $1249 \mathrm{~cm}^{-1}$. Anal. calcd for $\mathrm{C}_{18} \mathrm{H}_{19} \mathrm{Cl}-$ $\mathrm{N}_{2} \mathrm{O}_{2}$ : C 65.35, H 5.79, N 8.47; found C 65.46, H 5.43, N 5.87.

$O$-(2,4-二氯苯甲酰基)-3,3-二甲基-1-(吡啶-3-基)丁-2-酮肜(3k): 浅黄色油状物, 产率 93\%. [ $n]_{\mathrm{D}}^{20} 1.5796$. ${ }^{1} \mathrm{H} \mathrm{NMR}\left(400 \mathrm{MHz}, \mathrm{CDCl}_{3}\right) \delta: 1.28\left[\mathrm{~s}, 9 \mathrm{H}, \mathrm{C}\left(\mathrm{CH}_{3}\right)_{3}\right], 3.89$ (s, $2 \mathrm{H}, \mathrm{CH}_{2}$ ), $7.19 \sim 7.45$ (m, 5H, Py-H+Ph-H), 8.47 (s, $2 \mathrm{H}, \mathrm{Py}-\mathrm{H}) ;{ }^{13} \mathrm{C}$ NMR $\left(100 \mathrm{MHz}, \mathrm{CDCl}_{3}\right) \delta: 172.9,162.3$, $149.4,147.8,138.5,135.3,134.5,132.1,131.6,130.8$, $127.4,127.1,123.6,39.0,30.8,28.0$; IR (KBr) $v$ : 1758, $1623,1267 \mathrm{~cm}^{-1}$. Anal. calcd for $\mathrm{C}_{18} \mathrm{H}_{18} \mathrm{Cl}_{2} \mathrm{~N}_{2} \mathrm{O}_{2}$ : C 59.19, 
H 4.97, N 7.67; found C 59.32, H 4.82, N 7.57.

$O$-(3,4-二氯苯甲酰基)-3,3-二甲基-1-(吡啶-3-基)丁-2-酮肜 (3I): 白色固体，产率 94\%. m.p. 96.8 $97.3{ }^{\circ} \mathrm{C}$; ${ }^{1} \mathrm{H}$ NMR (400 MHz, $\left.\mathrm{CDCl}_{3}\right) \delta$ : $1.31[\mathrm{~s}, 9 \mathrm{H}$, $\mathrm{C}\left(\mathrm{CH}_{3}\right)_{3}$ ] $3.91\left(\mathrm{~s}, 2 \mathrm{H}, \mathrm{CH}_{2}\right), 7.23 \sim 7.70(\mathrm{~m}, 5 \mathrm{H}, \mathrm{Py}-\mathrm{H}+$ Ph-H), 8.50 (d, J=3.6 Hz, 1H, Py-H), 8.52 (s, 1H, Py-H); ${ }^{13} \mathrm{C}$ NMR (100 MHz, $\left.\mathrm{CDCl}_{3}\right) \delta: 172.9,161.7,149.3,148.1$, $137.9,134.9,133.0,131.5,131.2,130.7,128.6,128.3$, 123.6, 38.9, 30.7, 27.7; IR (KBr) $v: 1748,1619,1270 \mathrm{~cm}^{-}$ 1. Anal. calcd for $\mathrm{C}_{18} \mathrm{H}_{18} \mathrm{Cl}_{2} \mathrm{~N}_{2} \mathrm{O}_{2}$ : C 59.19, $\mathrm{H} 4.97, \mathrm{~N} 7.67$; found $\mathrm{C} 59.26, \mathrm{H} 4.73, \mathrm{~N} 7.64$.

\section{3 抑菌活性测试}

采用菌丝生长速率法测定目标化合物的离体抑菌 活性 ${ }^{[16]}$. 以茄子菌核菌 $(S$. sclerotiorum $)$ 和番茄灰霉菌 $(B$. cinerea)为测试菌种, 在预试验的基础上, 选择各供试化 合物生长抑制率在 10\% 90\%范围内的五个浓度梯度的 $\operatorname{PDA}($ 马铃薯一葡萄糖一琼脂培养基)含药平板, 并将其置 于培养血中固化. 将供试菌种的菌饼反接到含药平板中 央, 置于 $(25 \pm 1){ }^{\circ} \mathrm{C}$ 的培养箱中培养 $72 \mathrm{~h}$. 以加入等体 积无菌水的培养基为空白对照, 以杀菌剂三唑酮 (triadimefon)、百菌清(四氯间苯二腈, chlorothalonil)为对 照药剂. 每处理重复 3 次. 用十字交叉法测量菌落直径, 每个菌落测量 2 次, 以其平均值代表菌落的大小, 按以 下公式计算抑制率，最后通过(DPS)分析软件求出 $\mathrm{EC}_{50}$ 值. 试验结果见表 1 .

菌落扩散直径 $=$ 菌落平均直径一菌饼直径 抑制率 $(\%)=($ 对照菌落扩散直径一处理菌落扩散 直径 $) /($ 对照菌落扩散直径 $) \times 100 \%$.

\section{辅助材料(Supporting Information) 目标化合物 3a} 31 的 ${ }^{1} \mathrm{H} N M R$ 和 ${ }^{13} \mathrm{C}$ NMR 谱图. 这些材料可以免费从本 刊网站(http://sioc-journal.cn/)上下载.

\section{References}

[1] Zhang, Y.; Wang, B.-L.; Zhan, Y.-Z.; Zhang, L.-Y.; Li, Y.-H.; Li, Z.-M. Chem. J. Chin. Univ. 2016, 37, 1100 (in Chinese). (张燕, 王宝雷, 詹益周, 张丽媛, 李永红, 李正名, 高等学校化 学学报, 2016, 37, 1100.)

[2] Liu, W.-K.; Zhou, J.-P.; Zhang, T.; Zhu, H.-Y.; Qian, H.; Zhang, H.-B.; Huang, W.-L.; Gust, R. Bioorg. Med. Chem. Lett. 2012, 22, 2701.

[3] Chen, C.-X.; Wei, M.-X.; Li, X.-Q.; Li, T.-C.; Zhou, X.-Z. Chin. J. Org. Chem. 2015, 35, 835 (in Chinese). (陈凑喜, 魏梦雪, 李学强, 李天才, 周学章, 有机化学, 2015 , 35, 835.)

[4] Li, T.-X.; Zhang, J.; Pan, J.-K.; Wu, Z.-X.; Hu, D.-Y.; Song, B.-A. Eur. J. Med. Chem. 2017, 125, 657.

[5] Xu, T.-M.; Ji, W.-J.; Cai, F.; Xing, J.-H.; Kong, X.-L. Chin. J. Pestic. Sci. 2012, 14, 131 (in Chinese) (许天明，姬文娟，蔡飞，邢家华，孔小林，农药学学报，2012, 14, 131.)

[6] Dai, H.; Chen, J.; Hong, Y.; Yuan, B.-Y.; Chen, Y.-M.; Shi, Y.-J.; Ma, R.-Y.; Liang, Z.-P.; Shi, J. Chin. J. Org. Chem. 2017, 37, 739 (in Chinese).

(戴红, 陈佳, 洪宇, 袁斌颖, 陈雨蒙, 石玉军, 马瑞媛, 梁志鹏, 石健, 有机化学, 2017, 37, 739.)

[7] Guan, A.-Y.; Liu, C.-L.; Sun, X.-F.; Xie, Y.; Wang, M.-A. Bioorg. Med. Chem. 2016, 24, 342.

[8] Liu, X.-H.; Cui, P.; Song, B.-A.; Bhadury, P. S.; Zhu, H.-L.; Wang, S.-F. Bioorg. Med. Chem. 2008, 16, 4075

[9] Hwang, T.-L.; Wang, W.-H.; Wang, T.-Y.; Yu, H.-P.; Hsieh, P.-W. Bioorg. Med. Chem. 2015, 23, 1123.

[10] Li, N.; Li, Z.; Wang, K.-L.; Liu, Y.-X.; Li, Y.-Q.; Wang, Q.-M. Bioorg. Med. Chem. 2016, 24, 474.

[11] Karakurt, A.; Alagöz, M. A.; Sayoglu, B.; Çalıs, Ü.; Dalkara, S. Eur. J. Med. Chem. 2012, 57, 275.

[12] Liu, A.; Wang, X.; Chen, C.; Pei, H.; Mao, C.; Wang, Y.; He, H.; Huang, L.; Liu, X.; Hu, Z.; Ou, X.; Huang, M.; Yao, J. Pest. Manage. Sci. 2009, 65, 229

[13] Harini, S. T.; Kumar, H. V.; Rangaswamy, J.; Naik, N. Bioorg. Med. Chem. Lett. 2012, 22, 7588.

[14] Xia, L.-J.; Wang, X.-B.; Xie, Y.; Wang, X.-Y.; Xiao, W.; Zhong, X.-M.; Huang, M.-G.; Xue, W. Chin. J. Appl. Chem. 2017, 34, 316 (in Chinese).

(夏丽娟，王晓斌，谢艳，王晓燕，肖维，钟新敏，黄民国，薛伟， 应用化学, 2017, 34, 316.)

[15] Gedig, T.; Dettner, K.; Seifert, K. Tetrahedron 2007, 63, 2670.

[16] Sun, J.-L.; Mu, W. Pesticide Science Experimental Techniques and Guidance, Chemical Industry Press, Beijing, 2009, pp. 228 229 (in Chinese).

(孙家隆，慕卫，农药实验技术与指导，化学工业出版社，北京， 2009, pp. 228 229.)

(Li, L.; Fan, Y.) 